\title{
EXIT CHART AIDED IRREGULAR CONVOLUTIONAL CODE DESIGN FOR ITERATIVE DOWNLINK SDMA SYSTEMS USING IMPERFECT CHANNEL STATE INFORMATION
}

\author{
Chun-Yi Wei, Jin Wang and Lajos Hanzo \\ School of ECS., Univ. of Southampton, SO17 1BJ, UK. \\ Tel: +44-23-80-593 125, Fax: +44-23-80-593 045 \\ Email: lh@ecs.soton.ac.uk, http://www-mobile.ecs.soton.ac.uk
}

\begin{abstract}
We analyze a precoded and iteratively detected downlink multiuser system employing imperfect Spatio-Temporal Channel Impulse Response at the Transmitters (ST-CIRT) with the aid of Extrinsic Information Transfer (EXIT) charts. A Precoded Iterative DownLink Space Division Multiple Access (PI-DLSDMA) system proposed in our previous research is further improved and shown to be capable of maintaining an infinitesimally low BER, despite using imperfect ST-CIRT. A further novel feature of the PI-DL-SDMA system is that we design an IrRegular Convolutional Code (IRCC) with the aid of EXIT chart analysis for creating an open EXIT tunnel between the inner decoder's and outer decoder's EXIT curve at a reduced $E_{b} / N_{0}$ value and hence maintain an infinitesimally low BER.
\end{abstract}

Index Terms - SDMA, downlink, iterative, EXIT chart

\section{INTRODUCTION}

Extrinsic Information Transfer (EXIT) charts were proposed by ten Brink [1] and have been widely used for analyzing the extrinsic information exchange of iterative decoding aided systems. In the open literature, EXIT chart analysis has been typically used for systems exploiting the idealized simplifying assumption of having perfect Channel State Information (CSI), hence characterizing the achievable upper-bound performance of the systems analyzed. By contrast, in this treatise, we use EXIT charts for analyzing the achievable performance of the proposed system using realistic imperfect CSI and further improve the design of our system by taking the impact of imperfect CSI into account.

The system we considered is a precoded and iteratively detected downlink (DL) multiuser system [2], which we referred to a Precoded Iterative DownLink Space Division Multiple Access (PI-DL-SDMA) system. The transmitter of our system adopted the spatio-temporal DL pre-processing technique of [3] for eliminating the effects of Multi-User Interference (MUI), which may also be termed as a Multi-User

The financial support of the EU under the auspices of the Phoenix and Newcom project, as well as that the EPSRC UK is gratefully acknowledged.
Transmission (MUT) technique. The philosophy of this MUT is that in the presence of perfect knowledge of the individual users' Spatio-Temporal Channel Impulse Response (ST-CIR) to be encountered, the MUT scheme exploits the unique, userspecific ST-CIRs accurately differentiating the user's transmitted signals. Given that the MUT essentially eliminated the MUI, the low-complexity soft-out Minimum Mean Square Error (MMSE) detector of [4] may be invoked by the PI-DLSDMA multiuser system. Furthermore, a unity-rate convolutional encoder using a single shift register stage [5] is employed for precoding, because it was demonstrated in [2] that the performance of iterative decoding is substantially improved, when carried out by exchanging extrinsic information between the unity-rate precoder's decoder and the channel decoder.

Again, when the idealized scenario of having perfect knowledge of the ST-CIRs at the Transmitters (ST-CIRT) is assumed, our system employing the spatio-temporal pre-processing technique of [3] becomes capable of separating the signals destined for the different users at the base station's DL transmitter and hence results in MUI-free performance. In practice, the ST-CIRT has to be obtained by using realistic channel prediction algorithms $[6,7]$, which is particularly challenging for high throughput systems, where $(M \times N)$ numbers of ST-CIRs have to be estimated [4] for an $M$-element transmitter and $N$-element receiver. The resultant imperfect ST-CIRT will inflict an increased MUI upon each user's received signal and hence degrades the attainable performance of the system. Hence, we investigate the impact of imperfect ST-CIRT quantified in terms of the ST-CIRT prediction error variance, which adequately characterizes the quality of the ST-CIRT for a Gaussian error model. We will demonstrate using EXIT chart analysis that as expected, the area under the inner decoder's EXIT curve - where we refer to the MMSE detector concatenated with the precoder's decoder as the inner decoder - is reduced upon increasing the ST-CIRT prediction error variance, which may result in the closure of the decoder's open EXIT tunnel. More explicitly, once the inner decoder's EXIT curve crosses the outer decoder's owing to ST-CIRT errors, the system exhibits a high residual er- 
ror rate. In order to improve the achievable performance of our system proposed in [2] we design sophisticated IrRegular Convolutional Codes (IRCC) [8] using several different-rate component codes for producing a better-matching outer EXIT curve, which allows us to improve the attainable BER performance. More explicitly, the novel contribution of this treatise is that we propose an EXIT-chart based PI-DL-SDMA design technique using IRCCs, which allows us to take into account the expected level of ST-CIRT variance, hence resulting in an infinitesimally low BER, despite using imperfect channel prediction for MUT.

The rest of this treatise is structured as follows. In Section 2 we outline the system model used, while in Section 3 we summarize the design of IRCCs. Our EXIT chart analysis is provided in Section 4, leading to the performance results of Section 5. Finally, we conclude our discourse in Section 6.

\section{SYSTEM MODEL}

The structure of the PI-DL-SDMA system considered was described in [2]. Hence we only provide a brief summary of the system using Figure 1. More specifically, our system comprises a Base-Station (BS) employing $M$ transmit antennas for supporting $K$ Mobile Stations (MSs), where each of the MSs employs $N_{k}$ receive antennas. In this contribution we consider a flat-fading Multi-Input and Multi-Output (MIMO) channel. Consequently, each link between the $i$-th BS transmit antenna and the $j$-th MS receiver antenna of the $k$-th user may be characterized by a complex-valued scalar channel coefficient $H_{i j}^{(k)}$, which we assume to be an i.i.d. Gaussian random variable having a variance of unity and a mean of zero. Moreover, the MIMO channel corresponding to the $k$-th user may be described as an $\left(N_{k} \times M\right)$-dimensional complexvalued time-domain channel matrix $\mathbf{H}^{(k)}$, which may be defined as follows

$$
\mathbf{H}^{(k)}=\left(\begin{array}{cccc}
H_{1,1}^{(k)} & H_{1,2}^{(k)} & \cdots & H_{1, M}^{(k)} \\
H_{2,1}^{(k)} & H_{2,2}^{(k)} & \cdots & H_{2, M}^{(k)} \\
\vdots & \vdots & \cdots & \vdots \\
H_{N_{k}, 1}^{(k)} & H_{N_{k}, 2}^{(k)} & \cdots & H_{N_{k}, M}^{(k)}
\end{array}\right) .
$$

As illustrated in Figure 2, the data bits are encoded by both the channel encoder and the unity-rate precoder before modulation. More explicitly, the unity-rate precoder is a convolutional encoder using a single shift register stage [5]. Let $\mathbf{s}^{(k)} \in \mathbb{C}^{L_{k} \times 1}$ be a complex-valued column vector, which denotes the precoded data symbol vector to be transmitted to the $k$-th MS, while $L_{k}$ represents the number of independent data symbols contained in $\mathbf{s}^{(k)}$.

Additionally, we define the so-called space-time preprocessor matrix $\mathbf{T}^{(k)} \in \mathbb{C}^{M \times L_{k}}$, which was designed for the sake of eliminating the MUI [3]. As suggested by Choi and Murch in [3], we may formulate a solution of the MUT design problem as $\mathbf{T}^{(k)}=\mathbf{V}^{(k)} \mathbf{A}^{(k)}$, where $\mathbf{A}^{(k)}$ is a nonzero $\left(n_{k} \times L_{k}\right)$-dimensional matrix and $\mathbf{V}^{(k)}$ can be calculated using the SVD [9] of $\tilde{\mathbf{H}}^{(k)}$ expressed as:

$$
\tilde{\mathbf{H}}^{(k)}=\left(\begin{array}{cc}
\tilde{\mathbf{U}}^{(k)} & \mathbf{U}^{(k)}
\end{array}\right) \cdot\left(\begin{array}{cc}
\sum_{\mathbf{0}} & \mathbf{0} \\
\mathbf{0} & \mathbf{0}
\end{array}\right) \cdot\left(\begin{array}{c}
\tilde{\mathbf{V}}^{(k)^{H}} \\
\mathbf{V}^{(k)^{H}}
\end{array}\right),
$$

while

$$
\tilde{\mathbf{H}}^{(k)}=\left(\begin{array}{llllll}
\mathbf{H}^{(1)} & \ldots & \mathbf{H}^{(k-1)} & \mathbf{H}^{(k+1)} & \cdots & \mathbf{H}^{(K)}
\end{array}\right)^{T} .
$$

Furthermore, let $\mathbf{r}^{(k)}$ and $\mathbf{n}^{(k)}$ be the received signal vector and noise vector associated with the $k$-th MS, respectively. As it was demonstrated in [3], once the MUI was eliminated by the MUT, the received signal vector associated with the $k$-th MS can be expressed in the following form

$$
\mathbf{r}^{(k)}=\mathbf{H}^{(k)} \mathbf{T}^{(k)} \mathbf{s}^{(k)}+\mathbf{n}^{(k)},
$$

where the $\left(N_{k} \times L_{k}\right)$-dimensional matrix $\mathbf{H}^{(k)} \mathbf{T}^{(k)}$ characterizes the effective channel corresponding to the $k$-th MS.

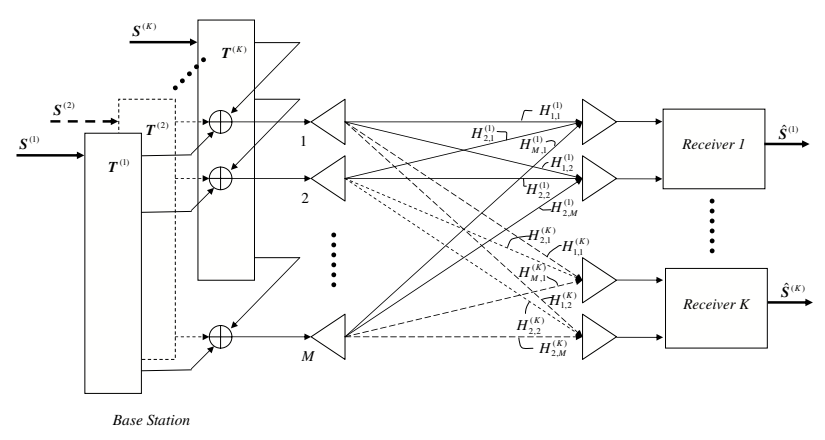

Fig. 1. Multiuser transmission in the DL-SDMA system

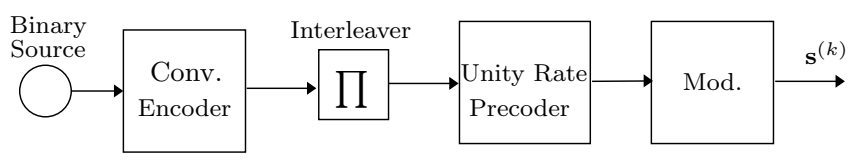

Fig. 2. Generating the precoded data symbols for the $k$-th user

As seen in Figure 3, the MMSE aided SDMA detector constitutes the first stage of the receiver. Iterative decoding is carried out by exchanging extrinsic information between the unity-rate precoder's decoder and the IRCC decoders. Figure 3 illustrates the iterative receiver's structure.

Let us now consider the impact of imperfect ST-CIRT. More specifically, let $\mathbf{H}_{\text {pred }}^{(k)}$ denote the predicted channel of the $k$-th MS, which is modeled as $\mathbf{H}_{\text {pred }}^{(k)}=\mathbf{H}^{(k)}+\varepsilon_{k}$, where 


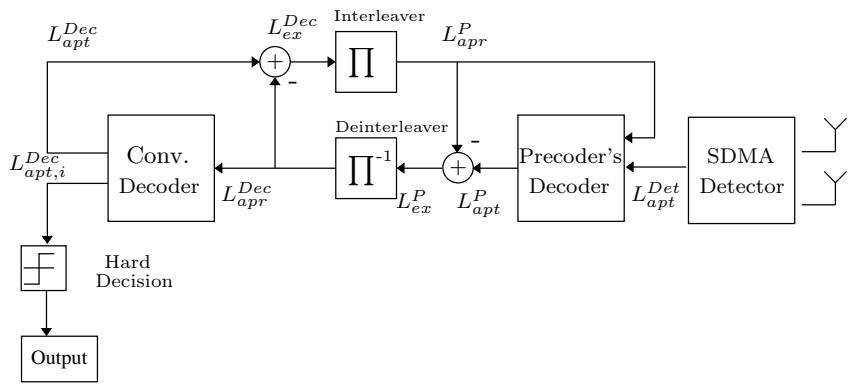

Fig. 3. Iteratively Decoded Receiver Design

$\varepsilon_{k}$ is assumed to be the Gaussian channel prediction error of the $k$-th MS. When the BS uses the predicted channel $\mathbf{H}_{\text {pred }}^{(i)}, i=$ $1,2, \cdots, k-1, k+1, \cdots, K$ to generate the space-time preprocessor matrix $\tilde{\mathbf{T}}^{(k)}$ for transmission to the $k$-th $\mathrm{MS}$, the system becomes unable to entirely eliminate the MUI and hence the resultant residual MUI contaminates the received signal of the $k$-th MS according to

$$
\begin{aligned}
\mathbf{r}^{(k)} & =\mathbf{H}^{(k)} \tilde{\mathbf{T}}^{(k)} \mathbf{s}^{(k)} \\
& +I_{M U I}\left(\varepsilon_{i}, i=1,2, \cdots, k-1, k+1, \cdots, K\right)+\mathbf{n}^{(k)},
\end{aligned}
$$

where the MUI term $I_{M U I}\left(\varepsilon_{i}, i=1,2, \cdots, k-1, k+1, \cdots, K\right)$ is a function of $\varepsilon_{i}$.

When using the space-time preprocessor matrix $\tilde{\mathbf{T}}^{(k)}$, no closed-form analytical formula is available for deriving the MUI in terms of the channel prediction error $\varepsilon_{i}$. Hence we used Monte Carlo simulations combined with semi-analytical EXIT charts to characterize the impact of channel prediction errors $\varepsilon_{i}$, which is assumed to be a Gaussian distributed random variable having a variance with $\sigma_{S T-C I R T}^{2}$ and a zero mean. Nonetheless, in order to establish a benchmarker, initially we assume that the receiver has perfect ST-CIRT.

\section{DESIGN OF IRREGULAR CONVOLUTIONAL CODES}

In order to design a near-capacity system, the outer decoder's EXIT chart has to match the inner decoder 's EXIT curve as accurately as possible, which results in an infinitesimally low EXIT-chart-tunnel area [10]. We employ IRCCs for solving this curve-fitting problem. Tüchler and Hagenauer $[8,11]$ proposed the employment of IRCCs, which are constituted by a family of convolutional codes having different code rates. They were specifically designed with the aid of EXIT charts, for the sake of improving the convergence behaviour of iteratively decoding systems. To be specific, an IRCC is constructed from a family of $P$ subcodes. First a rate- $r$ convolutional mother code $C_{1}$ is selected and the $(P-1)$ other subcodes $C_{j}$ of rate $r_{j}>r$ are obtained by puncturing. Let $l$ denote the total number of encoded bits generated from the $\kappa$ uncoded information bits. Each subcode encodes a certain fraction $\alpha_{j} r_{j} l$ of the original uncoded information bits and generates $\alpha_{j} l$ encoded bits. Given the target overall average code rate of $R \in[0,1]$, the weighting coefficient $\alpha_{j}$ has to satisfy:

$$
1=\sum_{j=1}^{P} \alpha_{j}, R=\sum_{j-1}^{P} \alpha_{j} r_{j}, \text { and } \alpha_{j} \in[0,1], \forall j .
$$

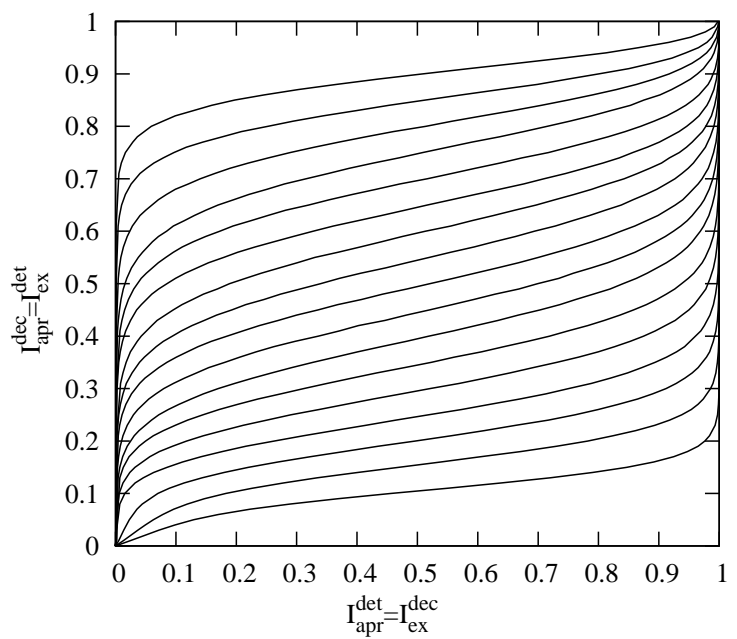

Fig. 4. EXIT functions of the 17 subcodes in [8]

For example, the EXIT functions of the 17 subcodes used in [8] are shown in Figure 4. In order to solve this curve-fitting problem, our optimized weighting coefficients are generated with the aid of the algorithm proposed by Tüchler and Hagenauer [8].

\section{EXIT CHART ANALYSIS FOR IMPERFECT ST-CIRT}

In this section, we provide the EXIT chart analysis of the PIDL-SDMA system in the presence of imperfect ST-CIRT. We adapt the IRCCs to match the inner decoder's EXIT curve in order to maintain a marginally open tunnel, which implies having both an infinitesimally low open-tunnel area as well as a low BER at the target $E_{b} / N_{0}$ value.

Figure 5 characterizes the impact of different ST-CIRT prediction error variances $\sigma_{S T-C I R T}^{2}$ on the system invoking the rate-0.5 $\operatorname{RSC}(2,1,3)$ channel decoder of [2] having the octal generator polynomials of $(5,7)$ and operating at an $E_{b} / N_{0}$ of $7 \mathrm{~dB}$. The value of $\sigma_{S T-C I R T}^{2}$ characterized in Figure 5 is ranging from 0 to 0.2 , where $\sigma_{S T-C I R T}^{2}=0$ represents a perfect ST-CIRT. Observe that when $\sigma_{S T-C I R T}^{2}$ increases, the corresponding EXIT curves of the inner decoder 
move closer to the curve of the $\operatorname{RSC}(2,1,3)$ outer channel decoder, potentially crossing the EXIT curve of the outer decoder. When the value of $\sigma_{S T-C I R T}^{2}$ is less than 0.1 , an open tunnel appears between the inner and outer EXIT curves. By contrast, the system fails to exhibit an open tunnel, when the value of $\sigma_{S T-C I R T}^{2}$ increases to 0.15 . Clearly, if the value

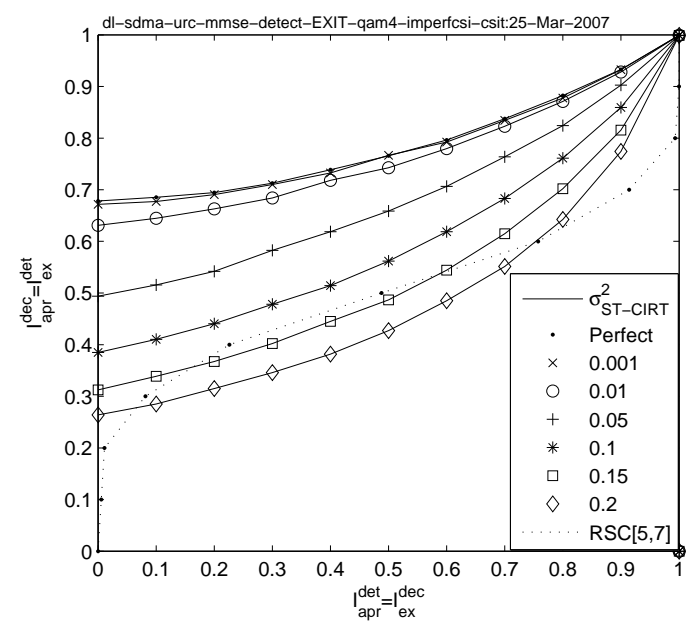

Fig. 5. EXIT chart analysis of the PI-DL-SDMA system employing rate- $0.5 \mathrm{RSC}(2,1,3)$ having the octal generator polynomials of $(5,7)$. The system operates at an $E_{b} / N_{0}$ of $7 \mathrm{~dB}$ and uses the parameters of Table 1 . The value of $\sigma_{S T-C I R T}^{2}$ assumes $0,0.001,0.01,0.05,0.1,0.15$ to 0.2 .

of $\sigma_{S T-C I R T}^{2}$ reaches 0.15 , it becomes necessary to arrive at a better-fitting outer curve. Hence, we employ the IRCCs briefly introduced in Section 3 in order to obtain a better fitting outer curve. Using the iterative algorithm of [8], we designed a rate- 0.5 IRCC employing the weighting coefficients $\alpha=[0,0,0,0,0,0,0.574825,0.167428,0,0,0,0.0291502$, $0.149874,0,0,0,0.0787566]$. The resultant EXIT chart is shown in Figure 6, which is seen to exhibit an open tunnel. The recorded iterative decoding trajectory seen in Figure 6 more accurately characterizes the convergence behavior of the system. When using $I=25$ iterations, the system employing a rate-0.5 IRCC exhibits an open tunnel, which implies that an infinitesimally low BER is expected at the $E_{b} / N_{0}=7 \mathrm{~dB}$. We will provide the corresponding BER performance results in the next section.

Figure 7 characterizes the convergence behavior of the system at $\sigma_{S T-C I R T}^{2}=0.1$, where the system employing the rate- 0.5 RSC exhibits a marginally open tunnel. The iterative decoding trajectory of the system employing the rate- 0.5 RSC was recorded using dotted lines in Figure 7 and requires $I=13$ iterations for approaching an infinitesimally low BER, because the EXIT-tunnel exhibits a constriction. By contrast, the recoded decoding trajectory of the system employing a rate- 0.5 IRCC requires only $I=7$ iterations for maintaining

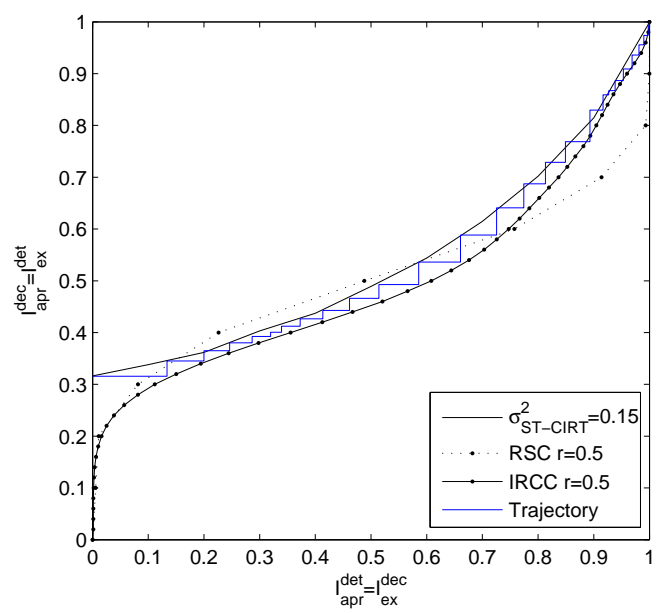

Fig. 6. EXIT chart analysis of the PI-DL-SDMA system employing rate-0.5 IRCC for $\sigma_{S T-C I R T}^{2}=0.15$. The system operates at an $E_{b} / N_{0}$ of $7 \mathrm{~dB}$ and uses the parameters of Table 1 .

Table 1. System Parameters

\begin{tabular}{|l|r|}
\hline Channel Encoder & $\begin{array}{r}\text { rate-0.5 RSC code } \\
\text { or rate-0.5 IRCC } \\
10^{5} \text { bits }\end{array}$ \\
Interleaver length & $4 \mathrm{QAM}$ \\
\hline Modulation & $K=3$ \\
\hline Number of users & $M=6$ \\
\hline Number of transmit antennas & \\
\hline $\begin{array}{l}\text { Dimension of transmitted } \\
\text { signal vector }\end{array}$ & $L_{k}=2$, for $k=1,2,3$. \\
\hline Number of receive antennas & $N_{k}=2$, for $\mathrm{k}=1,2,3$. \\
\hline
\end{tabular}

an open tunnel, as shown using solid lines, since the tunnel is more widely open.

\section{BER PERFORMANCE}

In this section, we provide the corresponding BER performance results, showing their reasonable consistency with our EXIT chart analysis. The system parameters used are listed in Table 1.

In Figure 6, we already showed that the rate-0.5 IRCC exhibits an open tunnel for the system having $\sigma_{S T-C I R T}^{2}=$ 0.15 . However, it is beneficial to explore the tolerable range of the ST-CIRT prediction variance of $\sigma_{S T-C I R T}^{2}$ for the system employing the rate- 0.5 IRCC. In Figure 8 , we portray the BER performance of the system operating at an $E_{b} / N_{0}$ of $7 \mathrm{~dB}$ for different ST-CIRT variances $\sigma_{S T-C I R T}^{2}$, ranging from 0.08 to 0.20 . As illustrated in Figure 8, the system employing the rate-0.5 IRCC and $I=25$ decoding it- 


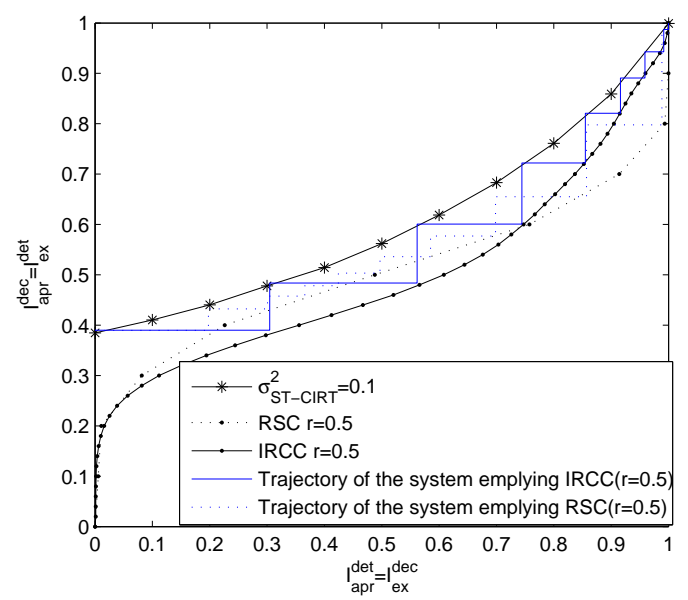

Fig. 7. The EXIT chart convergence behavior of the PI-DLSDMA system employing rate- $0.5 \mathrm{RSC}(2,1,3)$ and rate- 0.5 IRCC for $\sigma_{S T-C I R T}^{2}=0.1$. The system using the parameters of Table 1 operates at an $E_{b} / N_{0}$ of $7 \mathrm{~dB}$.

erations is capable of providing an infinitesimally low BER for $\sigma_{S T-C I R T}^{2}=0.15$. The BER performance of the system employing rate-0.5 RSC is also provided as a benchmarker. It appears that the system employing the rate- 0.5 RSC using $I=25$ decoding iterations exhibits an infinitesimally low BER for $\sigma_{S T-C I R T}^{2}=0.1$.

Figure 9 portrays the BER performance of the system employing the rate- 0.5 IRCC at different numbers of decoding iterations. We observed that when the number of decoding iterations increases, the system reaches an infinitesimally low BER for $\sigma_{S T-C I R T}^{2}=0.15$. This shows the consistency with Figure 6, which also exhibits an open tunnel for $I=25$.

Figure 10 portrays the attainable BER performance of the system employing the rate- 0.5 IRCC in conjunction with $\mathrm{I}=25$ decoding iterations for $\sigma_{S T-C I R T}^{2}=0.15$. While Figure 6 exhibits an open tunnel for $I=25$ and $\sigma_{S T-C I R T}^{2}=0.15$ at the target of $E_{b} / N_{0}=7 d B$, which implies an infinitesimally low BER for our system operating at an $E_{b} / N_{0}$ of at least $7 d B$, the results shown in Figure 10 demonstrate that an even lower $E_{b} / N_{0}$ is sufficient. We observed that the system employing the rate- 0.5 IRCC reaches an infinitesimally low BER at the $E_{b} / N_{0}$ value about $6.3 \mathrm{~dB}$. By contrast, the system employing a rate- 0.5 RSC exhibits a high BER in the excess of $10 \%$ at the same $E_{b} / N_{0}$.

\section{CONCLUSION}

In this contribution we investigated the impact of imperfect ST-CIRT on our PI-DL-SDMA system with the aid of EXIT charts. Furthermore, a novel IRCC scheme was designed for matching the outer decoder's EXIT curve to that of the in-

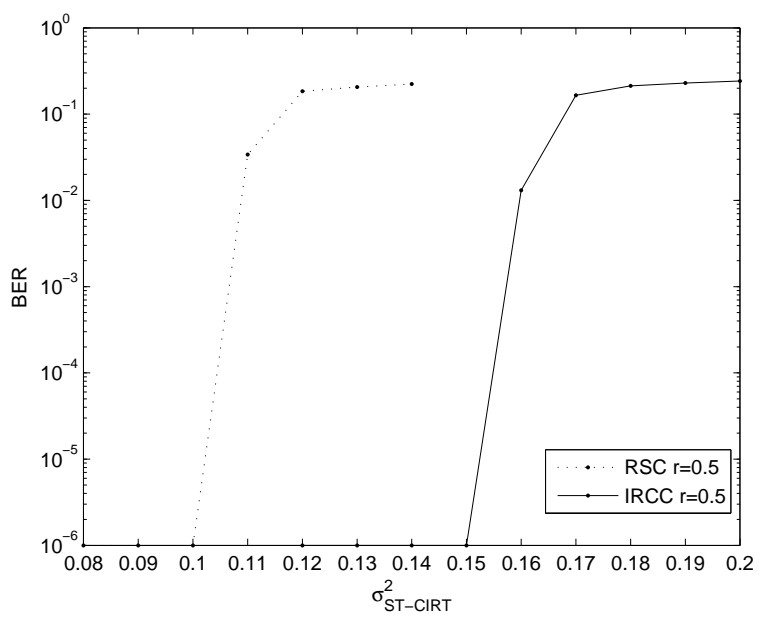

Fig. 8. BER performance of the PI-DL-SDMA system operating at an $E_{b} / N_{0}$ of $7 \mathrm{~dB}$ with different values of $\sigma_{S T-C I R T}^{2}$, ranging from 0.08 to 0.2 . The rate- 0.5 IRCC and rate- $0.5 \mathrm{RSC}$ are tested with the system, using 8 decoding iterations. The channel model was a flat fading MIMO channel and the parameters of Table 1 were used.

ner decoder and hence for minimizing the EXIT tunnels area, which allowed us to operate at a reduced $E_{b} / N_{0}$ value. In our future research we will use vector-quantized ST-CIRT signaling, combined with long-range channel prediction and highorder QAM.

\section{REFERENCES}

[1] S. ten Brink, "Designing iterative decoding schemes with the extrinsic information transfer chart," $A E \ddot{U}$ International Journal of Electronics Communications, vol. 55 , pp. 389 - 398, 2000.

[2] Chun-Yi Wei, Jos Akhtman, Soon Xin Ng, and Lajos Hanzo, "Iterative Near-Maximum-Likelihood Detection in Rank-Deficient Downlink SDMA Systems," IEEE Transactions on Vehicular Technology, accepted and scheduled to appear in the January 2008 issue.

[3] Lai-U Choi and R.D. Murch, "A transmit preprocessing technique for multiuser MIMO systems using a decomposition approach," IEEE Transactions on Wireless Communications, vol. 3, no. 1, pp. 20 - 24, 2004.

[4] L. Hanzo and M. Münster and B-J. Choi and T. Keller, OFDM and MC-CDMA for broadband multi-user communications, WLANs and Broadcasting, John Wiley and IEEE press, 2003.

[5] D. Divsalar, S. Dolinar, and F. Pollara, "Serial turbo trellis coded modulation with rate-1 inner code," 


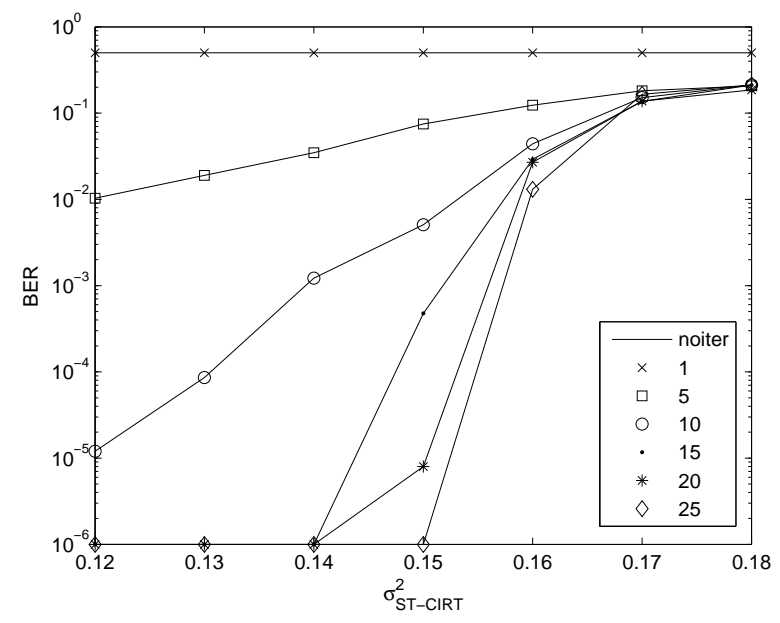

Fig. 9. BER performance of the PI-DL-SDMA system invoking rate- $0.5 \mathrm{IRCC}$ operating at an $E_{b} / N_{0}$ of $7 \mathrm{~dB}$ for different values of $\sigma_{S T-C I R T}^{2}$, ranging from 0.12 to 0.18 . The BER performance results of the system with different numbers of decoding iterations are compared. The channel model was a flat fading MIMO channel and the parameters of Table 1 were used.

IEEE Global Telecommunications Conference, 2000. GLOBECOM 'O0., vol. 2, pp. 777 - 782, 2000.

[6] Zhendong Luo, Hong Gao, Yuanan Liu, and Jinchun Gao, "Robust pilot-symbol-aided mimo channel estimation and prediction," IEEE Global Telecommunications Conference., vol. 6, pp. 3646-3650, 2004.

[7] A. Duel-Hallen, Shengquan Hu, and H. Hallen, "Longrange prediction of fading signals," IEEE Signal Processing Magazine, vol. 17, pp. 62-75, 2000.

[8] M. Tüchler and J. Hagenauer, "EXIT charts of irregular codes," in In Proceedings of Conference Information Sciences and Systems, Princeton [CDROM], NJ, USA, Mar, 2002.

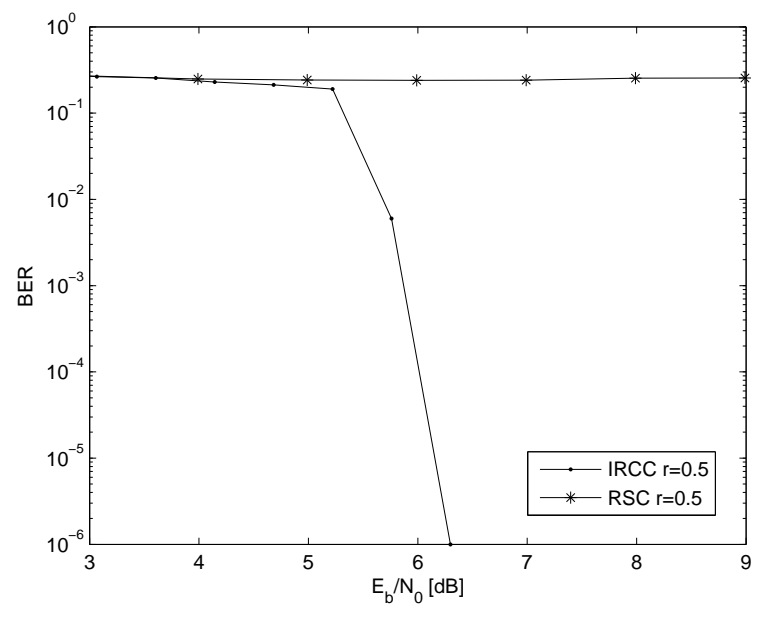

Fig. 10. BER performance of the PI-DL-SDMA system invoking the rate- $0.5 \mathrm{IRCC}$ using $\mathrm{I}=25$ decoding iterations for $\sigma_{S T-C I R T}^{2}=0.15$. The BER performance of the system invoking a rate $=0.5 \mathrm{RSC}$ using $\mathrm{I}=25$ decoding iterations is provided as a benchmarker. The channel model was a flat fading MIMO channel and the parameters of Table 1 were used.

[9] J. E. Gentle, Numerical linear algebra for applications in statistics, Springer-Verlag, Berlin, 1998.

[10] I. Land, P. Hoeher, and S. Gligorevic, "Computation of symbol-wise mutual information in transmission systems with $\log$ APP decoders and application to EXIT charts," Proceedings of the International ITG Conference on Source and Channel Coding (SCC), Erlangen, Germany, pp. 195 - 202, 2004.

[11] M. Tüchler, "Design of Serially Concatenated Systems Depending on the Block Length," IEEE Transactions on Communications, vol. 52, pp. 209-218, 2004. 